\title{
Análisis Causa-Raíz Sobre Evento Adverso Producido en la Clínica Odontológica Docente Asistencial, Facultad de Odontología, Universidad de La Frontera, Chile
}

\author{
Cause-Root Analysis on an Adverse Event Generated at the Dental Clinic, \\ Faculty of Dentistry, Universidad de La Frontera, Chile
}

\author{
Fernando Rivera-Mendoza',2; Claudia Acevedo-Atala1; Bernardo Perea-Pérez; \\ Elena Labajo-González ${ }^{3}$ \& Gabriel M. Fonseca ${ }^{1,4}$
}

RIVERA-MENDOZA, F.; ACEVEDO-ATALA, C.; PEREA-PÉREZ, B.; LABAJO-GonZÁLEZ, E. \& FONSECA, G. M. Análisis causa-raíz sobre evento adverso producido en la clínica odontológica docente asistencial, Facultad de Odontología, Universidad de la Frontera, Chile. Int. J. Odontostomat., 11(2):207-216, 2017.

RESUMEN: El Análisis de Causa-Raíz (ACR) es una forma de estudio retrospectivo de eventos adversos destinado a detectar las causas subyacentes de los mismos para proteger a los pacientes mediante la modificación de los factores dentro del sistema de salud que los provocaron y prevenir sus recurrencias. Si bien esta concepción centrada en la seguridad del paciente ha visto un importante auge en la atención médica, la odontología no ha sido llevada de igual manera probablemente por presentar daños más leves, procedimientos ambulatorios (con la consiguiente falta de seguimiento de muchos eventos adversos) y prácticas fundamentalmente privadas (cuyos conflictos afectarían potencialmente los resultados comerciales). Dado que no hay precedentes en Chile, se presenta un evento adverso producido en la Clínica Odontológica Docente Asistencial de la Facultad de Odontología de la Universidad de La Frontera y su ACR, desarrollado como primera intervención del Centro Chileno para la Observación y Gestión del Riesgo Sanitario de esa institución. Se plantean las necesidades de implementar un sistema explícito de categorización de eventos adversos en esa disciplina y de apoyar políticas de cultura en seguridad para el paciente odontológico, y se discute el papel de las instituciones universitarias para reconocer las áreas de vulnerabilidad en sus clínicas y así reforzar y mejorar la calidad de sus prácticas sanitarias.

PALABRAS CLAVE: análisis causa-raíz (ACR), odontología, educación odontológica, seguridad del paciente; reporte de eventos adversos, cultura sanitaria.

\section{INTRODUCCIÓN}

Los Eventos Adversos son "resultados no esperados de un tratamiento sanitario que provoca la prolongación del tratamiento, algún tipo de morbilidad, mortalidad o simplemente cualquier daño que el paciente no debería haber sufrido" (Perea-Pérez et al., 2011a). Este concepto es sumamente amplio e incluye errores, accidentes, retrasos asistenciales, negligencias, entre otros, pero no las complicaciones propias de la enfermedad del paciente (Perea-Pérez et al., 2011a). La detección de la causa subyacente de eventos que vulneren la seguridad del paciente, constituye la clave para minimizar el riesgo de que estos se repitan (Pandit \& Gong, 2016).
El Análisis de Causa-Raíz (ACR) es una forma de estudio retrospectivo de estos eventos para entender sus causas subyacentes mediante una revisión paso a paso de la cronología de los acontecimientos y de aquellos factores que permitan identificar su causal. Sobre la base de los campos de datos interoperables, un equipo organizado ACR puede investigar más a fondo el evento al revisar el informe, entrevistar al profesional o al paciente cuando lo considere necesario, y revisar las declaraciones de testigos, la ficha clínica y los protocolos de trabajo y procesos para determinar todas las posibles causas que podrían haber llevado a la producción del evento (Pandit \& Gong).

${ }^{1}$ Centro Chileno para la Observación y Gestión del Riesgo Sanitario, Facultad de Odontología, Universidad de La Frontera, Temuco, Chile.

${ }^{2}$ Programa de Magíster en Odontología, Facultad de Odontología, Universidad de La Frontera, Temuco, Chile.

${ }^{3}$ Observatorio Español para la Seguridad del Paciente Odontológico (OESPO), Departamento de Toxicología y Legislación Sanitaria, Escuela de Medicina Legal, Facultad de Medicina, Universidad Complutense, Madrid, España.

${ }^{4}$ Laboratorio de Pericias en Odontología Forense -LPO-, Facultad de Odontología, Universidad de La Frontera, Temuco, Chile. 
RIVERA-MENDOZA, F.; ACEVEDO-ATALA, C.; PEREA-PÉREZ, B.; LABAJO-GONZÁLEZ, E. \& FONSECA, G. M. Análisis causa-raíz sobre evento adverso producido en la clínica odontológica docente asistencial, facultad de odontología, universidad de la frontera, Chile. Int. J. Odontostomat., 11(2):207-216, 2017.

En consonancia con los estándares exigidos por el Ministerio de Salud de Chile para aseguramiento de la Calidad y Seguridad del paciente sanitario (Kraemer \& Vera, 2008; Ministerio de Salud, 2004; 2012), en Sesión Extraordinaria № 24 del 30 de marzo de 2016, el Consejo de Facultad de Odontología de la Universidad de La Frontera aprobó la creación del "Centro Chileno para la Observación y Gestión del Riesgo Sanitario", cuya misión es "minimizar la cantidad y calidad de eventos adversos inherentes a la actividad odontológica -por principio jurídico falible dada la naturaleza humana de quienes la realizan-, a través de la gestión adecuada del riesgo asistencial sanitario en la Facultad de Odontología de la Universidad de La Frontera". Entendiendo que entre las líneas de trabajo declaradas por este Centro se encuentra la "aplicación de herramientas diagnósticas: análisis retrospectivo de eventos adversos comunicados: ACR y análisis prospectivo de potenciales eventos adversos: Análisis Modal de Fallos y Efectos (AMFE)" (III. Organización y Administración del Centro Chileno para la Observación y Gestión del Riesgo Sanitario (OBSERVATORIO), punto C, inciso 3), se presenta un ACR sobre evento adverso producido en la Clínica Odontológica Docente Asistencial (C.O.D.A.) de esa casa de estudios, y se discute el conjunto de acciones relevantes para potenciar la educación en cultura de seguridad del paciente odontológico.

\section{REPORTE DE CASO}

Antecedentes. La Clínica Odontológica Docente Asistencial (C.O.D.A.) es una entidad dependiente de la Facultad de Odontología de la Universidad de La Frontera la que, junto a las instalaciones en el Centro de Diagnóstico y Tratamiento CDT del Hospital Henríquez Aravena de Temuco (Chile), ofrecen servicios de salud oral a la comunidad. La C.O.D.A. cuenta con 104 unidades dentales completas (sillones odontológicos), servicios de pabellón, equipos de imagenología y de esterilización para brindar atención odontológica a pacientes provenientes de distintas comunas de la Región de la Araucanía. Bajo ese concepto, estas instalaciones desarrollan una gran actividad docente a cargo de profesionales cirujanos dentistas supervisando permanentemente a más de 170 estudiantes de la Carrera de Odontología de esa universidad (http://odontologia.ufro.cl/ index.php/clinica-de-atencion).

El 6 de enero de 2016, fue recibido en la oficina de Contraloría de la institución el reclamo de una mu- jer de 66 años, paciente de la C.O.D.A., ingresada al sistema desde marzo de 2014 quien refiere la pérdida de un diente posteriormente a su tratamiento odontológico en el establecimiento, durante el cursado de la asignatura Clínica Odontológica Integral Adulto II (pregrado, $5^{\circ} \mathrm{Año)}$. La paciente manifiesta que "...luego de varias sesiones, donde fui atendida por un alumno en práctica que siempre me atendió de manera deferente y profesional, en la última de ellas el alumno, recibiendo instrucciones de la odontóloga a cargo, aplicó al parecer un procedimiento equivocado a $\mathrm{mi}$ modo de ver, lo que derivó en la pérdida del diente (sic). De acuerdo a lo anterior, solicito a ustedes, se me compense dicha pérdida con un tratamiento que permita corregir el daño provocado y a su vez eximir de responsabilidad al alumno que me atendió, toda vez que la responsable real del error es la odontóloga que supervisaba su trabajo".

En abril de ese año la oficina de Contraloría se expidió informando que la paciente "desdentada parcial con enfermedad periodontal y daño coronario en dientes 23 y 25 , recibió atención integral de tipo periodontal y rehabilitador en base a pulidos coronarios y prótesis fija en diente 23 y fallida prótesis fija en diente 25 , quedando sugeridos tratamiento de prótesis parcial superior. A la fecha la paciente presenta resto radicular de mal pronóstico en diente 25 . El paciente solicita mejora en tratamiento de ese diente. Se sugiere realizar exodoncia de resto radicular de diente 25 y posteriormente prótesis parcial removible superior, todo esto sin costo para paciente...". El 26 de abril de ese año, la Coordinadora Clínica de la C.O.D.A. consiente la resolución de Contraloría y paralelamente, solicitó al OBSERVATORIO la realización de ACR para la evaluación de los hechos, factores colaboradores, evento adverso presentes en este caso y mecanismos de prevención del riesgo.

Metodología ACR. El objetivo de realizar un ACR es proteger a los pacientes mediante la identificación y modificación de los factores dentro del sistema de salud que potencialmente pueden conducir a daño. Para el trabajo de campo se siguieron las fases estandarizadas por la National Patient Safety Foundation (2016) ajustadas a la secuencia propuesta por Charles et al. (2016), y según los lineamientos propuestos para odontología por Perea-Pérez et al. (2011b):

Paso 1. Identificación del Evento Adverso. Desde la creación del OBSERVATORIO, la Facultad de Odontología de la UFRO lleva a cabo una importante tarea 
de fomento para el reporte honesto y voluntario del Evento Adverso con acceso a una plantilla de reporte en la página oficial de la institución (http:// odontologia.ufro.cl). Debido al carácter inicialmente judicializado del evento, la Coordinadora Clínica de la C.O.D.A. tomó en consideración la realización del ACR, con lo que la Oficina de Contraloría entregó al OBSERVATORIO toda la información disponible respecto de Ficha Clínica y documentación basal del caso presentado.

Paso 2. Organización del Equipo. El OBSERVATORIO cuenta con un Director (Doctor en Odontología, experto en Odontología Legal), acompañado por un Comité Asesor de cuatro académicos (Doctores,
Magister y Especialistas odontólogos) administrando respectivas áreas específicas (Diagnóstica y Terapéutica; Rehabilitación y Reconstrucción; Odontopediatría; Postgrado e Investigación) y cuatro técnicos (odontólogos y administrativos de la C.O.D.A.). Este Comité permanente solicita a su vez la participación de expertos según el caso en tratamiento, siempre considerando que cada uno de los miembros no se encuentre involucrado directamente en el caso, para asegurar objetividad (Charles et al.). El tiempo de realización del ACR demandó aproximadamente unos 45 días.

Paso 3. Desarrollo de un Diagrama Inicial de Flujo. La Fig. 1 representa esquemáticamente los hechos conocidos:

\begin{tabular}{|c|c|c|c|}
\hline $\begin{array}{l}\text { 1. Unidad de Diagnóstico } \\
\text { (UI) evaluó al caso con } \\
\text { una complejidad apta } \\
\text { para ser abordado en el } \\
\text { pregrado. }\end{array}$ & $\begin{array}{l}\text { 2. Un año después se } \\
\text { retomó tratamiento sin } \\
\text { nueva evaluación de UI. } \\
\text { Se actualizó diagnóstico: } \\
\text { diente con fracturas. }\end{array}$ & $\begin{array}{l}\text { 3. El estudiante originó } \\
\text { una falsa vía durante la } \\
\text { desobturación. Esto no se } \\
\text { señaló en ficha (A). }\end{array}$ & $\begin{array}{l}\text { 4. Se realizaron pruebas, } \\
\text { ajuste y cementado final } \\
\text { de perno de fibra. Se } \\
\text { inició protocolo de } \\
\text { tallado para PFS. }\end{array}$ \\
\hline
\end{tabular}
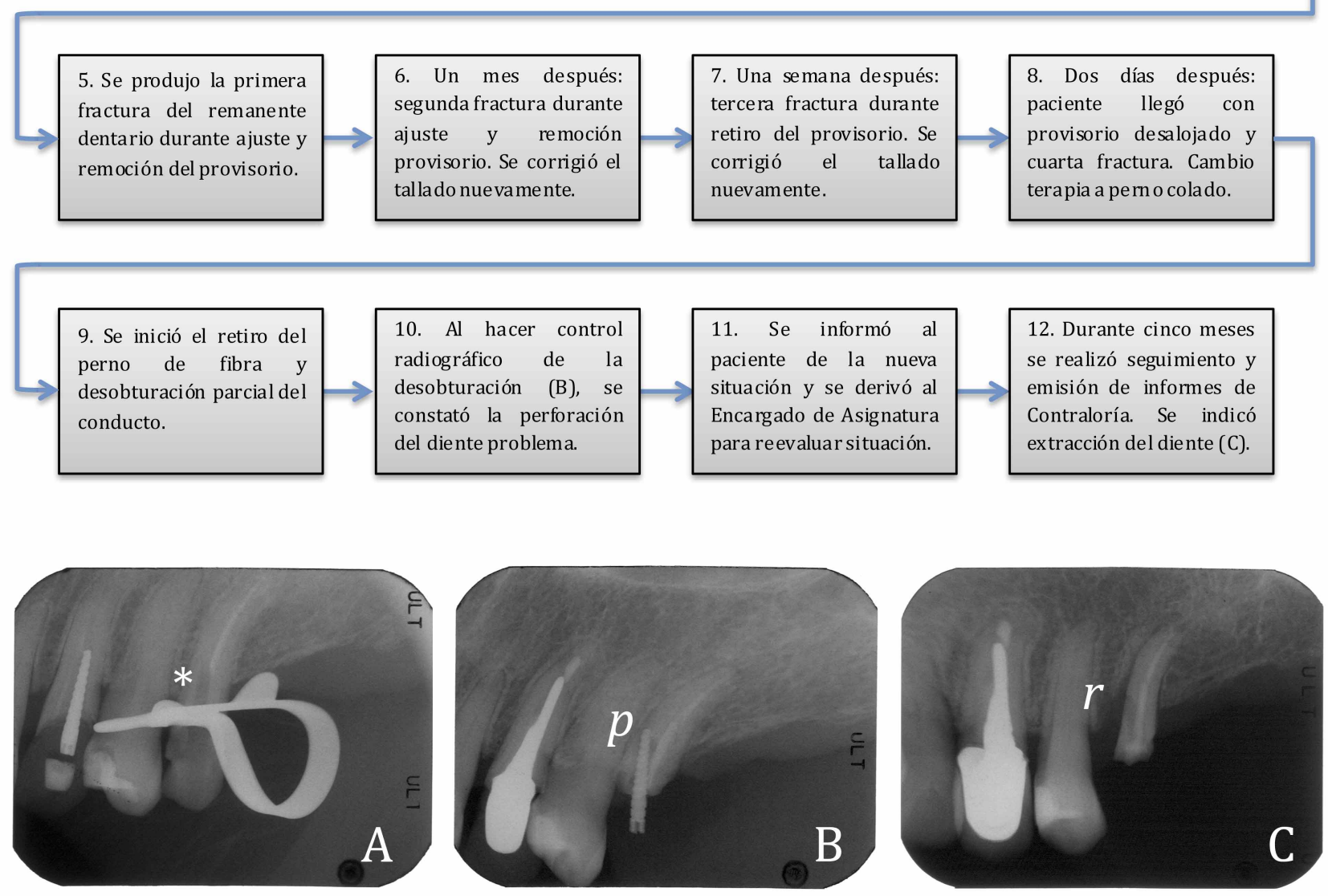

Fig. 1. Diagrama de flujo inicial y radiografías presentando los hechos conocidos. A: Radiografía periapical del día 20 de octubre de 2015. Nótese la falsa vía producida durante la desobturación parcial del conducto en el diente $2.5\left(^{*}\right)$. B: Radiografía periapical del día 21 de diciembre de 2015. Nótese la perforación lateral $(p)$ del diente problema y su coincidencia con la falsa vía producida dos meses antes. C: Radiografía periapical control del día 29 de febrero de 2016. La gran pérdida de sustancia y el proceso de reabsorción ósea ( $r$ ) en relación a la perforación llevaron a la indicación de extracción dentaria (adaptado de National Patient Safety Foundation). 
RIVERA-MENDOZA, F.; ACEVEDO-ATALA, C.; PEREA-PÉREZ, B.; LABAJO-GONZÁLEZ, E. \& FONSECA, G. M. Análisis causa-raíz sobre evento adverso producido en la clínica odontológica docente asistencial, facultad de odontología, universidad de la frontera, Chile. Int. J. Odontostomat., 11(2):207-216, 2017.

En marzo de 2014, la mujer fue citada para su evaluación por la Unidad de Diagnóstico de la C.O.D.A., la que determinó el grado de complejidad del caso luego de revisar los antecedentes, su condición clínica y exámenes imagenológicos complementarios. Sobre esa determinación, la paciente fue ingresada al sistema para su evaluación clínica y diagnóstica integral por un estudiante de quinto año bajo supervisión docente en Clínica Integral Adulto II. Durante el transcurso de ese año, el estudiante realizó las acciones de alta básica y periodontal, postergando la fase de rehabilitación planificada para el siguiente año.

A fines de marzo de 2015, la paciente retomó su tratamiento con el mismo estudiante, quien actualizó el diagnóstico y la planificación. Se consignó al diente 25 con "fractura coronaria asintomática, observando en radiografía relleno completo" e indicando una prótesis fija singular (PFS), previamente realizando una obturación provisional como parte de las acciones del tratamiento básico.

Entre los meses de julio a octubre de 2015, e iniciando el proceso de preparación de PFS, el estudiante realizó la desobturación parcial del relleno endodóntico y la confección de obturaciones provisionales con vidrio ionómero. Una vez finalizada la desobturación parcial, se realizó la prueba de perno fibra 0,5 con buen ajuste, el cual se cementó de manera definitiva con cemento autoadhesivo universal de resina. Se realizó obturación con composite y pulido.
El 9 de noviembre de 2015, se realizó el tallado del 25. Durante la confección del provisorio, este se sobreajustó al remanente dentario y en el proceso de retiro, se fracturó. Los procesos de rectificación del tallado y reajuste de provisorios se realizarían en las siguientes tres sesiones.

El 9 de diciembre de 2015, y nuevamente durante el retiro del provisorio, el remanente volvió a fracturarse. Se reconstituyó con composite, se talló, para luego ser reajustado y cementado de manera provisoria.

El 16 de diciembre de 2015 se repitió la secuencia de fractura al momento del retiro del provisorio. Nuevamente se reconstituyó con composite, se volvió a tallar, y el provisorio se reajustó y cementó.

El 18 de diciembre de 2015 la paciente llegó con el provisorio desalojado y una nueva fractura en el diente. En vistas de este nuevo escenario, se decidió cambiar el tratamiento de perno fibra a perno colado. El cambio de acción quedó consignado en la ficha solo con la firma del Jefe de Clínica. El provisorio se ajustó y cementó con vidrio ionómero.

El 21 de diciembre de 2015, se retiró el perno fibra y se desobturó el conducto. En el control radiográfico se detectó perforación lateral, con lo que la paciente fue informada de la imposibilidad de continuar la PFS. La paciente afirmó entender lo sucedido y fue derivada al Profesor Encargado de la Asignatura para evaluar la situación clínica y la conducta a seguir.

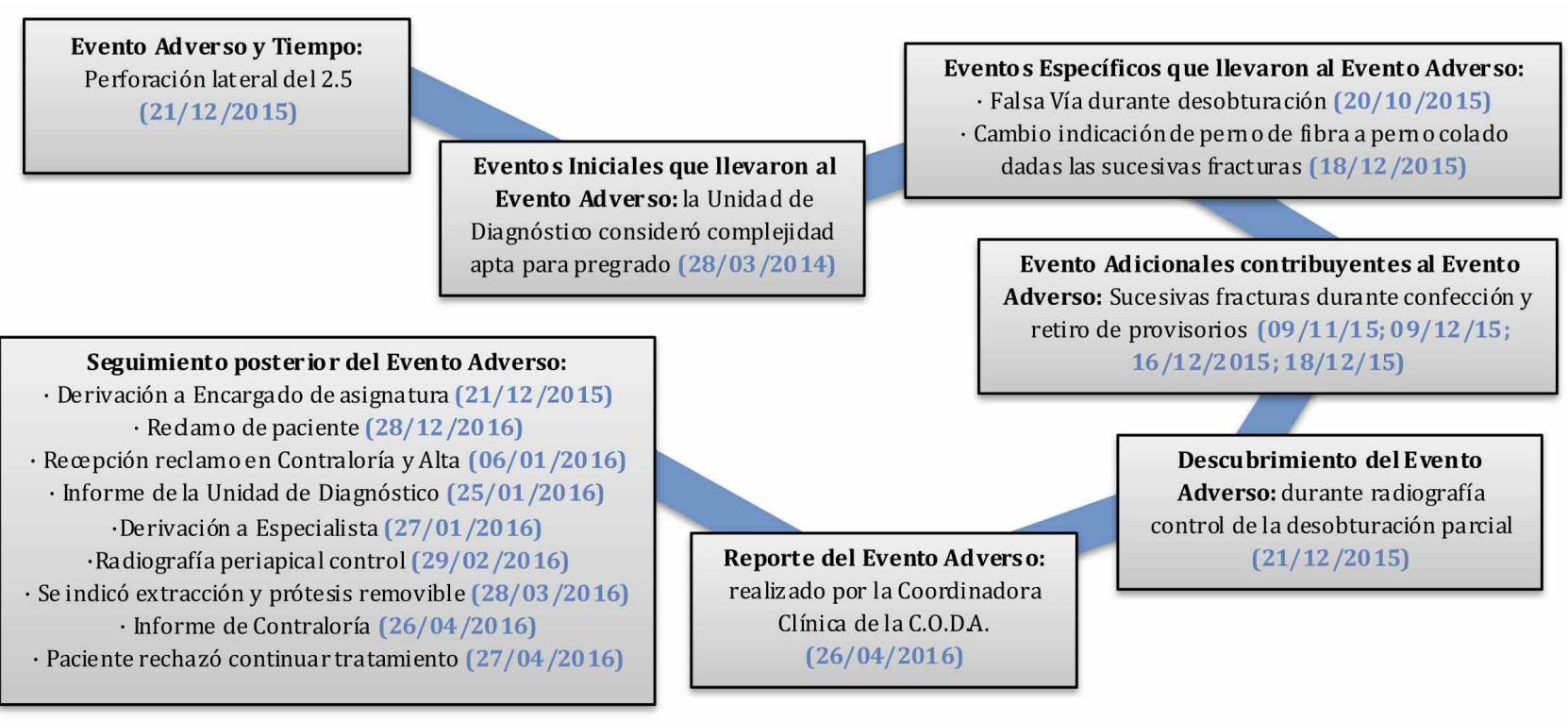

Fig. 2. Mapa de Reconstrucción de los Hechos del caso presentado (adaptado de Charles et al.). 
RIVERA-MENDOZA, F.; ACEVEDO-ATALA, C.; PEREA-PÉREZ, B.; LABAJO-GONZÁLEZ, E. \& FONSECA, G. M. Análisis causa-raíz sobre evento adverso producido en la clínica odontológica docente asistencial, facultad de odontología, universidad de la frontera, Chile. Int. J. Odontostomat., 11(2):207-216, 2017.

Paso 4. Desarrollo de un Mapa de Reconstrucción de los Hechos. Una vez realizadas las "preguntas gatillo", ayudas cognitivas para proporcionar el contexto clínico apropiado para interpretar lo ocurrido (Charles et al.), se expuso de manera gráfica la información recopilada a partir de la ficha y de las entrevistas a los actores asistenciales del evento adverso (Fig. 2).
Paso 5. Desarrollo de un Diagrama de Causa y Efecto. Este diagrama se encuentra compuesto por una declaración de problema, una acción y dos o tres condiciones (Charles et al.). Estas categorías deben involucrar problemas comunicacionales, administrativos, procedimientos y errores humanos conducentes al evento. Cada evento deberá estar conectado a una

Tabla I. Eventos, puntos críticos, barreras y estrategias para reducción de riesgos.

\begin{tabular}{|c|c|c|c|}
\hline Eventos & Puntos críticos & Barreras & Estrategias para reducción de riesgos \\
\hline $\begin{array}{l}\text { EVENTO INICIAL: } \\
\text { Unidad de Diagnóstico } \\
\text { consideró que el caso } \\
\text { era apto en su } \\
\text { complejidad para ser } \\
\text { abordado en el } \\
\text { pregrado }\end{array}$ & $\begin{array}{l}\text { Competencias del estudiante } \\
\text { de pregrado para evaluar } \\
\text { complejidad del caso } \\
\text { Paciente reinicia un año } \\
\text { después tratamiento sin } \\
\text { nueva evaluación } \\
\text { diagnóstica. El diente } \\
\text { presenta fracturas. }\end{array}$ & $\begin{array}{c}\text { Protocolo de evaluación } \\
\text { clínica para derivación pre y } \\
\text { postgrado } \\
\text { (línea roja) }\end{array}$ & 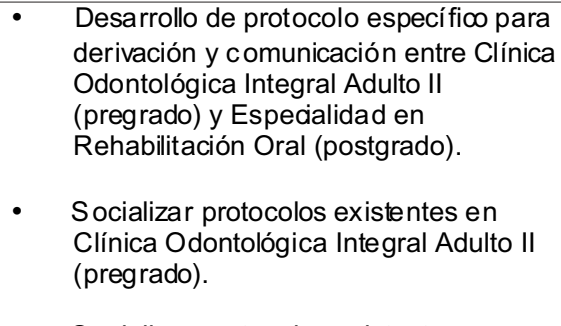 \\
\hline $\begin{array}{l}\text { EVENTO } \\
\text { ESPECÍFICO: El } \\
\text { estudiante desobtura } \\
\text { el diente problema, } \\
\text { originando una falsa } \\
\text { vía. Esto no es } \\
\text { señalado en }\end{array}$ & $\begin{array}{c}\text { Rectificación en proceso de } \\
\text { desobturación de relleno } \\
\text { endodóntico }\end{array}$ & $\begin{array}{l}\text { Establecer límite de } \\
\text { complejidad: curvatura } \\
\text { radicular } \\
\text { (línea roja) }\end{array}$ & $\begin{array}{l}\text { - Socializar protocolos existentes en } \\
\text { Clínica Odontológica Integral Adulto II } \\
\text { (pregrado). } \\
\text { - Supervisión efectiva del docente } \\
\text { instructor y Jefe de Clínica. } \\
\text { - Socializar protocolos existentes en } \\
\text { Clínica Odontológica Integral Adulto II } \\
\text { (pregrado). } \\
\text { Supervisión efectiva del docente } \\
\text { instructor y Jefe de Clínica. }\end{array}$ \\
\hline la ficha clínica & $\begin{array}{l}\text { Paciente no informada de } \\
\text { riesgos y evolución de } \\
\text { tratamiento }\end{array}$ & $\begin{array}{l}\text { Información a la paciente y } \\
\text { consentimiento firmado en } \\
\text { ficha clínica }\end{array}$ & $\begin{array}{l}\text { - } \quad \text { Consentimiento informado Ad-hoc. } \\
\text { - Lista de chequeo }\end{array}$ \\
\hline $\begin{array}{c}\text { EVENTO } \\
\text { ADICIONAL: } \\
\text { Fracturas de } \\
\text { remanente dentario } \\
\text { inmediatas a retiros }\end{array}$ & $\begin{array}{l}\text { Maniobras imprudentes } \\
\text { durante el proceso de } \\
\text { confección y/o retiro de } \\
\text { provisorio }\end{array}$ & $\begin{array}{l}\text { Protocolo para confección y } \\
\text { cementación de provisorios } \\
\text { en rehabilitación mediante } \\
\text { PFS }\end{array}$ & $\begin{array}{l}\text { - Socializar protocolos existentes en } \\
\text { Clínica Odontológica Integral Adulto II } \\
\text { (pregrado). } \\
\text { - Supervisión efectiva del docente } \\
\text { instructor y Jefe de Clínica. }\end{array}$ \\
\hline $\begin{array}{c}\text { y/o confecciones de } \\
\text { provisorio }\end{array}$ & $\begin{array}{l}\text { Paciente no informada de } \\
\text { riesgos y evolución de } \\
\text { tratamiento } \\
\text { Ficha no controlada }\end{array}$ & $\begin{array}{l}\text { Información a la paciente y } \\
\text { consentimiento firmado en } \\
\text { ficha clínica }\end{array}$ & $\begin{array}{l}\text { - } \quad \text { Consentimiento informado Ad-hoc. } \\
\text { - Lista de chequeo }\end{array}$ \\
\hline & $\begin{array}{l}\text { (inconsistencia en historial } \\
\text { previo endodóntico, cantidad } \\
\text { y morfología de canal(es), } \\
\text { referencias y tipos de } \\
\text { cemento utilizados) }\end{array}$ & $\begin{array}{l}\text { Seguir conductas regulares } \\
\text { para control de fichas por } \\
\text { instructor y Jefe de Clínica }\end{array}$ & $\begin{array}{l}\text { - } \quad \text { Supervisión efectiva del docente } \\
\text { instructor y Jefe de Clínica. } \\
\text { - } \quad \text { Lista de chequeo }\end{array}$ \\
\hline & $\begin{array}{l}\text { Paciente no informada de } \\
\text { riesgos y evolución de } \\
\text { tratamiento }\end{array}$ & $\begin{array}{l}\text { Información a la paciente y } \\
\text { consentimiento firmado en } \\
\text { ficha clínica }\end{array}$ & $\begin{array}{l}\text { - } \quad \text { Consentimiento informado Ad-hoc. } \\
\text { Lista de chequeo }\end{array}$ \\
\hline \multirow[t]{2}{*}{$\begin{array}{l}\text { EVENTO ADVERSO: } \\
\text { Perforación lateral } \\
\text { durante remoción del } \\
\quad \text { pemo de fibra }\end{array}$} & $\begin{array}{l}\text { Cambio curso terapéutico de } \\
\text { perno de fibra a colado }\end{array}$ & $\begin{array}{c}\text { Límites de acción según } \\
\text { curvatura, remoción, } \\
\text { debilidad del diente, entre } \\
\text { otros } \\
\text { (línea roja) }\end{array}$ & $\begin{array}{l}\text { - Socializar protocolos existentes en } \\
\text { Clínica Odontológica Integral Adulto II } \\
\text { (pregrado). } \\
\text { - Supervisión efectiva del docente } \\
\text { instructor y Jefe de Clínica. } \\
\text { - Socializar protocolos existentes en } \\
\text { Clínica Odontológica Integral Adulto II } \\
\text { (pregrado). }\end{array}$ \\
\hline & Retiro de perno de fibra & $\begin{array}{l}\text { Protocolo para retiro de } \\
\text { pernos de fibra. Apoyo o } \\
\text { derivación a especialista } \\
\text { rehabilitador o endodoncista }\end{array}$ & $\begin{array}{l}\text { - Supervisión efectiva del docente } \\
\text { instructor y Jefe de Clínica. } \\
\text { Desarrollo de protocolo específiœ para } \\
\text { derivación y comunicación entre Clínica } \\
\text { Odontológica Integral Adulto II } \\
\text { (pregrado) y Especialidad en } \\
\text { Rehabilitación Oral (postgrado). }\end{array}$ \\
\hline
\end{tabular}


RIVERA-MENDOZA, F.; ACEVEDO-ATALA, C.; PEREA-PÉREZ, B.; LABAJO-GONZÁLEZ, E. \& FONSECA, G. M. Análisis causa-raíz sobre evento adverso producido en la clínica odontológica docente asistencial, facultad de odontología, universidad de la frontera, Chile. Int. J. Odontostomat., 11(2):207-216, 2017.

causa precedente mediante una designación "causado por". Este proceso se continúa hasta agotar el conocimiento del evento poniendo en evidencia las investigaciones adicionales que fueran necesarias. Este diagrama pretende ayudar al equipo a identificar los vínculos causales (Fig. 3). Los errores humanos no deberán ser considerados nunca como una CausaRaíz aceptable (National Patient Safety Foundation).

Paso 6. Identificación de los Factores Contribuyentes de la Causa-Raíz (FCCR). Siguiendo las 5 reglas de la causalidad para la elaboración de declaraciones FCCR (Charles et al.), se detectaron falencias y errores comunicacionales-organizacionales: inconsistencias en la comunicación entre el docente instructor y el Jefe de Clínica; falta en la ejecución de un protocolo ante las reiteradas fracturas del remanente dental y los ajustes de provisorios. También se detectó en la ficha la ausencia de registro de las conductas a seguir y de lo que hubiera sido una oportuna derivación a especialistas, todos elementos que finalmente aportaron y/o contribuyeron al origen y/o desarrollo del evento adverso que generó el reclamo de la paciente. Se identificaron además barreras de comu- nicación con la paciente, al no existir constancias de haber sido informada del estado previo riesgoso del diente problema (estaba desvitalizado y con historial de fracturas anteriores), de las complicaciones generadas en el transcurso del tratamiento, o de la naturaleza del evento producido y sus posibles consecuencias.

Paso 7. Desarrollo de acciones correctivas. La Tabla I presenta el evento adverso, los eventos iniciales, específicos y adicionales contribuyentes, así como también las barreras y las estrategias para la reducción de riesgos para evitar que la Causa-Raíz vuelva a producirse.

Paso 8. Medidas de resultados. La Tabla II expone las acciones de mejora, los responsables de efectivizarlas, el tiempo estimado requerido para su seguimiento y el sistema por el cual se evaluarán.

Paso 9. Comunicación de resultados. El OBSERVATORIO convocó a una Mesa de Discusión donde se presentó la evaluación realizada sobre el caso. Fue convocada la coordinación clínica de C.O.D.A, dos

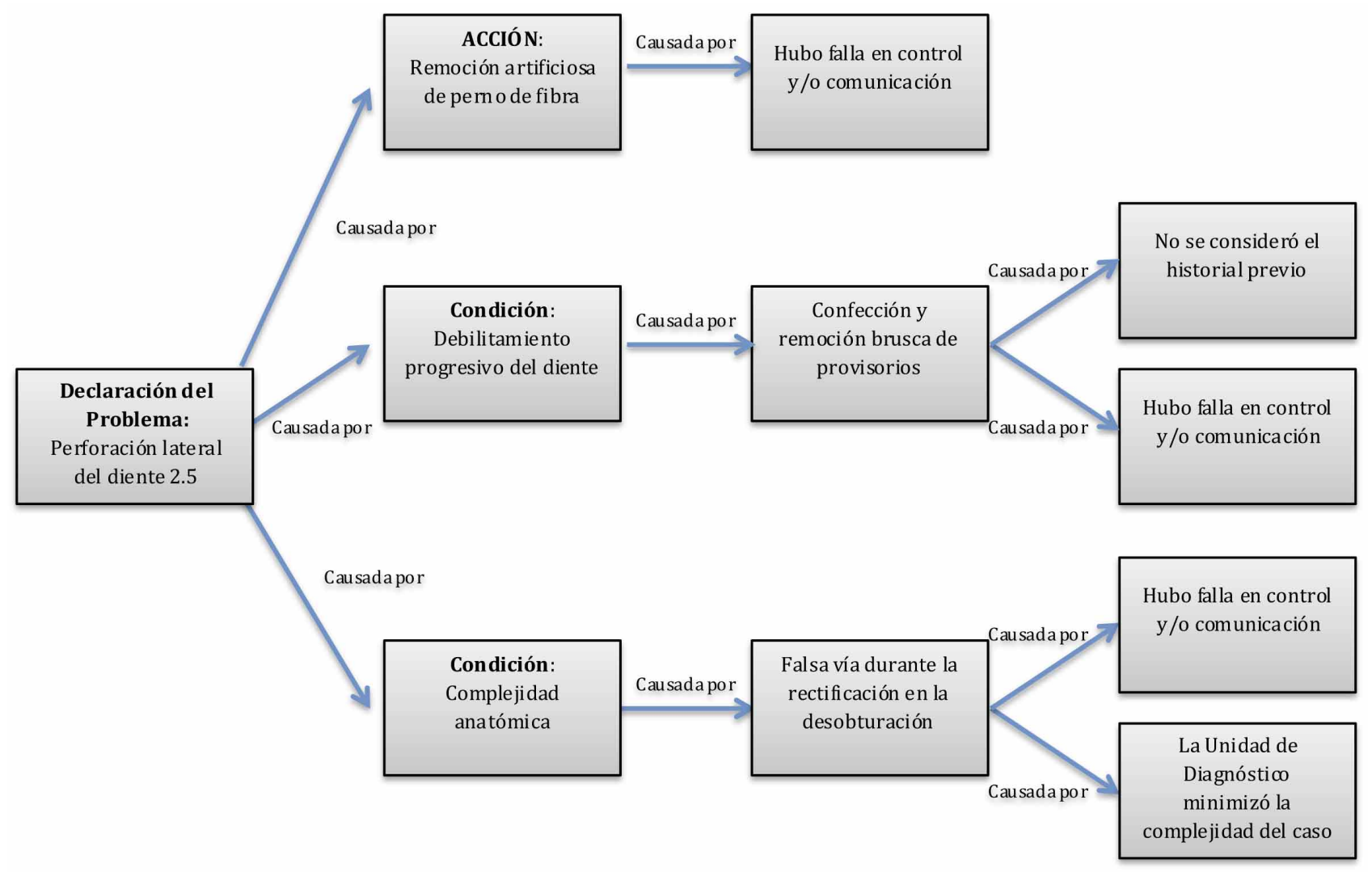

Fig. 3. Diagrama de Causa y Efecto para el caso presentado (adaptado de National Patient Safety Foundation, 2016). 
RIVERA-MENDOZA, F.; ACEVEDO-ATALA, C.; PEREA-PÉREZ, B.; LABAJO-GONZÁLEZ, E. \& FONSECA, G. M. Análisis causa-raíz sobre evento adverso producido en la clínica odontológica docente asistencial, facultad de odontología, universidad de la frontera, Chile. Int. J. Odontostomat., 11(2):207-216, 2017.

especialistas en el área de rehabilitación, el docente instructor protagonista del evento y el equipo del OBSERVATORIO. El Jefe de Clínica no respondió a la consigna. Esta Mesa consensuó las acciones de mejora y destacó el papel jugado por la Unidad de Diagnóstico al minimizar la complejidad del caso; el punto crítico correspondió a las competencias apropiadas del estudiante de pregrado para realizar tal tarea, y la ne- cesidad de establecer un protocolo específico con líneas rojas claras entre los parámetros y competencias de atención clínica entre pre y postgrado. Finalmente, se consensuó la presencia de inconsistencia en los registros clínicos en los dos años que estuvo bajo tratamiento la paciente. En fecha 23 de agosto de 2016, el OBSERVATORIO remitió el correspondiente informe a la Coordinadora Clínica de la C.O.D.A.

Tabla II. Acciones de mejora, responsabilidad, plazos e instrumento de evaluación de las mismas.

\begin{tabular}{|c|c|c|c|}
\hline Acciones de Mejora & Responsable & $\begin{array}{l}\text { Tiempo estimado } \\
\text { requerido }\end{array}$ & $\begin{array}{l}\text { Sistema de } \\
\text { Evaluación }\end{array}$ \\
\hline $\begin{array}{l}\text { Protocolización en seguridad del } \\
\text { paciente al equipo docente clínico }\end{array}$ & $\begin{array}{ll}\text { - } & \text { Coordinadora Clínica C.O.D.A. } \\
\text { Centro Chileno para la } \\
\text { Observación y Gestión del } \\
\text { Riesgo Sanitario }\end{array}$ & 6 meses & $\begin{array}{l}\text { Desarrollo de Listas de } \\
\text { chequeo }\end{array}$ \\
\hline $\begin{array}{l}\text { Modificación del Consentimiento } \\
\text { Informado }\end{array}$ & $\begin{array}{l}\text { - } \quad \text { Coordinadora Clínica C.O.D.A. } \\
\text { Centro Chileno para la } \\
\text { Observación y Gestión del } \\
\text { Riesgo Sanitario }\end{array}$ & 6 meses & $\begin{array}{l}\text { Consentimiento } \\
\text { Informado Ad-hoc }\end{array}$ \\
\hline Modificación de ficha clínica & $\begin{array}{l}\text { - } \quad \text { Coordinadora Clínica C.O.D.A. } \\
\text { Centro Chileno para la } \\
\text { Observación y Gestión del } \\
\text { Riesgo Sanitario }\end{array}$ & 1 año & $\begin{array}{l}\text { Ficha clínica } \\
\text { multidimensionada }\end{array}$ \\
\hline Control efectivo de fichas clínicas & $\begin{array}{ll}\text { - } & \text { Instructores. } \\
\text { - } & \text { Jefes de Clínica } \\
\text { Encargados de Asignatura }\end{array}$ & 1 mes & $\begin{array}{l}\text { Supervisión efectiva } \\
\text { responsable de } \\
\text { asignatura }\end{array}$ \\
\hline $\begin{array}{l}\text { Protocolización de manejo ante } \\
\text { accidentes clínicos en rehabilitación }\end{array}$ & $\begin{array}{l}\text { Equipo docente Clínica } \\
\text { Odontológica Integral Adulto II } \\
\text { (pregrado) y Especialidad en } \\
\text { Rehabilitación Oral (postgrado) } \\
\text { - } \quad \text { Mesas de Discusión } \\
\text { Centro Chileno para la } \\
\text { Observación y Gestión del } \\
\text { Riesgo Sanitario }\end{array}$ & 1 año & Protocolos específicos \\
\hline
\end{tabular}

\section{DISCUSIÓN}

Se ha mencionado que los recursos para identificar, analizar y remediar las amenazas a la seguridad sanitaria no son ilimitados, con lo que es esencial utilizar un sistema explícito de priorización basado en el riesgo para que una organización pueda determinar de manera creíble y eficiente qué peligros deben abordarse primero: dentro de las 72 horas posteriores a la ocurrencia del evento, el primer paso será asignar una puntuación o categoría que determine la severidad del mismo, esto basado en la extensión de la lesión, la duración de la estadía, el nivel de cuidado requerido para su solución y los costos reales o estimados a asumir por la institución. Para esto, se han propuesto las categorías Menor (paciente sin lesión, sin aumento de la estadía ni aumento del nivel de atención, con daños o pérdidas económicas menores o inferiores a 10.000 dólares), Moderado (paciente con aumento de la estadía o del nivel de atención, con pérdidas económicas entre los 10.000 y los 100.000 dólares), Mayor (pacientes con disminución menor permanente de funciones corporales no relacionadas con el curso natural de la enfermedad o consecuentes a actos de comisión u omisión, o lesiones desfigurantes, que requieran intervención quirúrgica, aumento de la estadía, o pérdidas económicas mayores a los 100.000 dólares) y Catastrófico (pacientes fallecidos o con disminución permanente importante de funciones corporales no relacionadas con el curso natural de la enfermedad o consecuentes a actos de comisión u omisión, resultados directos de una caída, asociados con salidas no autorizadas y tratamien- 
RIVERA-MENDOZA, F.; ACEVEDO-ATALA, C.; PEREA-PÉREZ, B.; LABAJO-GONZÁLEZ, E. \& FONSECA, G. M. Análisis causa-raíz sobre evento adverso producido en la clínica odontológica docente asistencial, facultad de odontología, universidad de la frontera, Chile. Int. J. Odontostomat., 11(2):207-216, 2017.

tos interrumpidos, o resultado de asaltos $u$ otros crímenes) (National Patient Safety Foundation). Este sistema de triage pretenderá evaluar el reporte del evento para determinar si el ACR es justificable (Charles et al.), y si ese es el caso, iniciar la revisión del caso tan pronto como sea posible para poder capturar los detalles mientras aún están en mente de los involucrados (National Patient Safety Foundation). No hemos detectado algún tipo de implementación de ACR en instituciones odontológicas chilenas, menos aún sistemas de jerarquización de eventos adversos específicamente para este tipo de atención sanitaria; si bien el caso que se presenta probablemente justificó su tratamiento ACR sólo por las pretensiones económicas del reclamo de la paciente (el Laboratorio de Pericias en Odontología Forense -LPO- de la misma institución, de manera paralela llevó a cabo una valoración del daño para estimar los gastos emanados para una potencial indemnización, elemento no contemplado en el presente reporte), es evidente que se hace necesaria la implementación de un sistema de priorización de riesgo y categorización del evento adverso odontológico (estableciendo la pertinencia o no de un ACR), aplicable explícitamente para la C.O.D.A. y con potencial uso en otras instituciones sanitarias de esa especialidad. El caso que se presenta, siendo el primero reportado en la odontología chilena (y la primera intervención formal del OBSERVATORIO) justificó en la "mecánica de entrenamiento" que supuso.

Se ha mencionado que el "error humano" no es una causa-raíz aceptable pues, de estar involucrado en un evento adverso, su misma existencia implica que puede volver a ocurrir. Es fundamental comprender cuáles son los factores sistémicos que facilitaron ese error, y entender que disciplinando o formando al proveedor puede reducir la probabilidad de que el evento se repita con dicho proveedor, pero no frente a otro en circunstancias similares. Las soluciones dirigidas directamente al error humano (tareas de rehabilitación, capacitación e implementación de normas) son todas soluciones débiles y no deben utilizarse para evaluar desempeños individuales ni para disciplinar, avergonzar o castigar al personal (National Patient Safety Foundation).

Los Análisis de Causa-Raíz (ACR) son una aproximación sistemática orientada a descubrir las causas de incidentes y eventos adversos para establecer medidas preventivas. Los equipos ACR inves- tigan más allá del error humano para identificar problemas en el sistema que contribuyan o que resulten en la producción de estos eventos. El éxito radica en lograr responder ¿qué sucedió?, ¿por qué sucedió?, y ¿qué puede hacerse para evitar que suceda de nuevo? (Charles et al.). Si bien el caso presentado no reúne los elementos de complejidad ni la trascendencia clínica habitualmente requeridos para desarrollar un ACR (Charles et al.; Perea-Pérez et al., 2011b), la judicialización de la situación planteada desde el inicio por la paciente llevaron a poner en marcha este proceso desde el OBSERVATORIO para responder a las necesidades implícitas de identificar la concurrencia de eventos adversos y la prevención de sus recurrencias. No hay precedentes (cuando menos, formales) de una trabajo de estas características en instituciones odontológicas chilenas y, de hecho, el modelo adoptado por el OBSERVATORIO pretende replicar el desarrollado por el Observatorio Español para la Seguridad del Paciente Odontológico (OESPO), organismo creado en 2009 por el Consejo General de Odontólogos y Estomatólogos de España y la Universidad Complutense de Madrid para "fomentar la seguridad del paciente odontológico y prevenir cualquier tipo de riesgo derivado de la asistencia dental" (http://www.ucm.es/toxlegal/oespo). Aunque se han señalado limitaciones en los alcances de un ACR (susceptibilidad a politización de su gestión, tendencia a producir controles insuficientes de riesgo, malfuncionamiento de su retroalimentación, confusión sobre culpa y responsabilidad, etc.) (Peerally et al., 2016), y en odontología específicamente existen muy escasas referencias a su implementación técnica (Peleg et al., 2010; PereaPérez et al., 2011b), coincidimos con la literatura en la necesidad de investigación y reportes para comprender a cabalidad cuáles son las claves para llevar adelante de manera correcta una atención sanitaria odontológica centrada en el paciente que asegure calidad, seguridad y eficiencia (Mills et al., 2013; Ramoni et al., 2012; 2014).

Se ha afirmado que los profesionales de la salud poseen dificultades para asimilar eventos adversos cuando estos ocurren, esto originado en una cultura de "infalibilidad" que asume los errores como un defecto que debe ser ocultado y un marco legal adverso a la investigación de estos conflictos (Chomali \& Miranda, 2003). Esta centralización en el actuar del profesional (lex artis vs. malpraxis) originada en un reclamo (legítimo o no) por parte del paciente ha construido un modelo punitivo sumamente confuso, reactivo y extemporáneo donde las deci- 
RIVERA-MENDOZA, F.; ACEVEDO-ATALA, C.; PEREA-PÉREZ, B.; LABAJO-GONZÁLEZ, E. \& FONSECA, G. M. Análisis causa-raíz sobre evento adverso producido en la clínica odontológica docente asistencial, facultad de odontología, universidad de la frontera, Chile. Int. J. Odontostomat., 11(2):207-216, 2017.

siones médicas son tomadas más por temor que por motivos clínicos o preventivos (Chomali \& Miranda). Sin embargo, el enfoque de la atención sanitaria ha variado considerablemente en los últimos años centrándose esencialmente en el paciente y en la preocupación de no producirle daños (Perea-Pérez et al., 2011a). Este nuevo modelo ha logrado mejorar los resultados con mayor satisfacción tanto para el paciente como para el profesional, disminuyendo además la cantidad de litigios y reclamos por esta atención. Este modelo centralizado en el paciente es una medida fundamental de calidad en atención sanitaria (Mills et al.).

En el ámbito odontológico esta propuesta no ha sido llevada de igual manera, probablemente por presentar daños más leves, procedimientos ambulatorios (con la consiguiente falta de seguimiento de muchos eventos adversos) y prácticas fundamentalmente privadas (cuyos conflictos afectarían potencialmente los resultados comerciales) (PereaPérez et al., 2011a; Ramoni et al., 2014). Sin embargo, algo tan razonable como "aprender de los errores y difundir los conocimientos adquiridos" (PereaPérez et al., 2011b) parece echarse en falta en esta disciplina, donde la ausencia de políticas de cultura en seguridad del paciente no es un dato menor, y ha sido convenientemente mencionada (Perea-Pérez et al., 2011a; Ramoni et al., 2014; Fonseca et al., 2015).

Para Castillo (2016), aunque las metas internacionales de seguridad del paciente no han sido diseñadas per se para el ámbito odontológico, cada una de ellas puede ser gestionada identificando el momento oportuno de su aplicación y, más importante aún, fomentando una cultura de calidad desde las mismas entidades responsables de la formación del recurso humano: las facultades de odontología. Sin embargo, se ha informado que, desde sus servicios clínicos como escenarios de capacitación efectiva, ellas aún adolecen de necesidades de mejoramiento en cultura de seguridad del paciente (Leong et al., 2008; Ramoni et al., 2014). Específicamente en Chile, la educación superior odontológica se ha visto afectada por la desregulación originando modelos al arbitrio del mercado sin mayores exigencias de calidad (Alcota et al., 2016). Sólo 11 de 39 programas de odontología en ese país tienen sus programas acreditados, con currículas en su mayoría no adecuadas al contexto social y programas desarticulados entre pre y postgrado. El resultado es la presencia de proyectos educativos heterogéneos $y$, muy probablemente, profesionales odontólogos que no logran responder a las demandas sociales o que no son capaces de asumir la gestión del cuidado de las personas proporcionando cuidados de calidad, seguros y costo-efectivos (Alcota et al.). Sobre este punto, ya ha sido mencionado el riesgo implícito de entrelazar provisión de salud con mecanismos de enseñanza-aprendizaje en futuros profesionales: los pacientes se transforman en simples objetos de enseñanza, con lo que cualquier procedimiento realizado en clínicas odontológicas universitarias parece justificarse en nombre de enseñanza. El escenario es algo caótico desde esta perspectiva, con modelización de criterios donde se tipifica al paciente, priorización de metas y no de calidad, o demoras en los procedimientos se ven justificadas dada la naturaleza del efector (el estudiante y su proceso de aprendizaje)... Dicho de otra manera: promoción singular de eventos adversos (Finkler et al., 2015).

Las Facultades de Odontología representan el sostén fundamental de la salud oral de un país, no sólo como instituciones educativas aportando la mano de obra calificada, sino también como centros de descubrimiento asegurando que la práctica odontológica evolucione mediante la investigación y la transferencia de los últimos avances científicos. Desde esta perspectiva, y quizás como una de las misiones más sensibles, la provisión de cuidado oral configura una misión distintiva de las instituciones académicas dentales que ha evolucionado desde la atención clínica casual a un modelo centrado en el paciente replicando los ofrecidos por las facultades de medicina. La responsabilidad de la gestión sanitaria recae así en estas instituciones con contribuciones más eficientes y focalizadas a la gestión de sus pacientes (ADEA, 2016). Coincidimos con la literatura en que estos entes académicos deben asumir el desafío de reconocer sus áreas de vulnerabilidad como una oportunidad de fortalecer su propia cultura de seguridad para el paciente odontológico. Ya que las facultades de odontología entrenan futuras generaciones de cirujanos dentistas en el mundo, ellas mismas deben revisar sus actuales programas de enseñanza clínica, educar a su personal docente, estudiantes y facultativos respecto de las necesidades de monitoreo y reporte de sus incidentes y eventos adversos, así como también motivar investigación adicional para reforzar las mejores prácticas para seguridad de sus pacientes (Leong et al.).

Al fin y al cabo, las universidades deben asumir el rol de representar el estándar de oro de lo que se enseña en sus actividades clínicas. 
RIVERA-MENDOZA, F.; ACEVEDO-ATALA, C.; PEREA-PÉREZ, B.; LABAJO-GONZÁLEZ, E. \& FONSECA, G. M. Análisis causa-raíz sobre evento adverso producido en la clínica odontológica docente asistencial, facultad de odontología, universidad de la frontera, Chile. Int. J. Odontostomat., 11(2):207-216, 2017.

RIVERA-MENDOZA, F.; ACEVEDO-ATALA, C.; PEREAPÉREZ, B.; LABAJO-GONZÁLEZ, E. \& FONSECA, G. M. Cause-Root Analysis on an adverse event generated at the dental clinic, Faculty of Dentistry, Universidad de La Frontera, Chile. Int. J. Odontostomat., 11(2):207-216, 2017.

ABSTRACT: Root cause analysis (RCA) is a retrospective study of adverse events performed to detect the underlying causes of these events to protect patients by modifying the factors within the health system that caused them and preventing their recurrences. Although this paradigm focused on patient safety has seen a significant increase in medical care, dentistry has not been carried out in the same way, probably because of milder injuries, outpatient procedures (with the consequent lack of followup of many adverse events) and basically private practices (whose conflicts would potentially affect commercial outcomes). Since there is no precedent in Chile, we present an adverse event produced at the Dental Clinic of the Faculty of Dentistry of the University of La Frontera and its RCA, performed as the first intervention of the Chilean Center for the Observation and Management of Health Risk of that institution. The needs to implement an explicit system of categorization of adverse events in this discipline and to provide support for cultural safety policies for the dental patient are discussed. The role of university institutions in recognizing areas of vulnerability in their clinics and to strengthen and improve the quality of their health practices is also discussed.

KEY WORDS: root cause analysis (RCA), dentistry, dental education, patient safety, adverse event reporting, safety culture.

\section{REFERENCIAS BIBLIOGRÁFICAS}

ADEA Position Paper: Statement on the Roles and Responsibilities of Academic Dental Institutions in Improving the Oral Health Status of All Americans (As approved by the 2004 ADEA House of Delegates). J. Dent. Educ., 80(7):884-91, 2016.

Alcota, M.; Ruiz de Gauna, P. \& González, F. E. Dental programs in the current context of chilean higher education. Int. J. Odontostomat., 10(1):85-91, 2016.

Castillo, H. P. C. Seguridad del paciente en los servicios de estomatología. Rev. ADM, 73(3):155-62, 2016.

Charles, R.; Hood, B.; Derosier, J. M.; Gosbee, J. W.; Li, Y.; Caird, M. S., Biermann, J. S. \& Hake, M. E. How to perform a root cause analysis for workup and future prevention of medical errors: a review. Patient Saf. Surg., 10:20, 2016.

Chomali, G. M. \& Miranda, S. F. Gestión de riesgos en la atención de salud: Hacia una cultura de la calidad basada en la seguridad. Rev. Clin. Las Condes, 14(4), 2003. Disponible en: http:// www.elsevier.es/es-revista-revista-medica-clinica-las-condes202-pdf-X071686400331994X-S300

Finkler, M.; Caetano, J. C. \& Souza Ramos, F. R. Formación ética profesional: El paternalismo del vínculo profesor-alumno en la relación clínica. Eidón, (44):43-5, 2015.
Fonseca, G. M.; Mira, K.; Beltrán, H.; Peña, K. \& Yendreka, V. Eventos adversos y demandas por mala praxis en endodoncia. Int. J. Med. Surg. Sci., 2(1):367-75, 2015.

Kraemer, P. \& Vera, L. A. Revisión de Estrategias Efectivas para la Seguridad de la Atención del Paciente. Santiago de Chile, Ediciones Ministerio de Salud de Chile, 2008.

Leong, P.; Afrow, J.; Weber, H. P. \& Howell, H. Attitudes toward patient safety standards in U.S. dental schools: a pilot study. J. Dent. Educ., 72(4):431-7, 2008.

Ministerio de Salud. Ley 19.937. Modifica el D.L. No 2.763 de 1979, con la Finalidad de Establecer una Nueva Concepción de la Autoridad Sanitaria, Distintas Modalidades de Gestión y Fortalecer la Participación Ciudadana. Santiago de Chile, Gobierno de Chile, 2004.

Ministerio de Salud. Ley 20.584. Regula los Derechos y Deberes que tienen las Personas en Relación con Acciones Vinculadas a su Atención en Salud. Santiago de Chile, Gobierno de Chile, 2012.

Mills, I.; Frost, J.; Moles, D. R. \& Kay, E. Patient-centred care in general dental practice: sound sense or soundbite? Br. Dent. J., 215(2):81-5, 2013.

National Patient Safety Foundation. RCA2 Improving Root Cause Analyses and Actions to Prevent Harm. Second Online Publication, Version 2. The Joint Commission, 2016. Disponible en https://www.jointcommission.org/sentinel_event.aspx

Pandit, S. \& Gong, Y. Event reports promoting root cause analysis. Stud. Health Technol. Inform., 225:452-6, 2016.

Peerally, M. F.; Carr, S.; Waring, J. \& Dixon-Woods, M. The problem with root cause analysis. B. M. J. Qual. Saf., 26(5):417-22, 2016.

Peleg, O.; Givot, N.; Halamish-Shani, T. \& Taicher, S. Wrong tooth extraction: root cause analysis. Quintessence Int., 41(10):86972, 2010

Perea-Pérez, B.; Santiago-Sáez, A.; García-Marín, F.; LabajoGonzález, E. \& Villa-Vigil, A. Patient safety in dentistry: dental care risk management plan. Med. Oral Patol. Oral Cir. Bucal, 16(6):e805-9, 2011a.

Perea-Pérez, B.; Santiago-Sáez, A. \& Labajo-González, E. Análisis causa-raíz (ACR) de un evento adverso en odontología: inyección de una solución de hipoclorito sódico. Cient. Dent., 8(1):27-34, 2011b.

Ramoni, R.; Walji, M. F.; Tavares, A.; White, J.; Tokede, O.; Vaderhobli, R. \& Kalenderian, E. Open wide: looking into the safety culture of dental school clinics. J. Dent. Educ., 78(5):74556, 2014.

Ramoni, R. B.; Walji, M. F.; White, J.; Stewart, D.; Vaderhobli, R.; Simmons, D. \& Kalenderian, E. From good to better: toward a patient safety initiative in dentistry. J. Am. Dent. Assoc., 143(9):956-60, 2012.

Dirección para correspondencia:

Prof. Dr. Gabriel M. Fonseca

Centro Chileno para la Observación y Gestión del Riesgo Sanitario

Facultad de Odontología

Universidad de La Frontera

Temuco - CHILE

E-mail: gabriel.fonseca@ufrontera.cl 